\title{
Políticas públicas educativas sobre migración en los institutos de secundaria de España
}

\section{Public education policies on migration in secondary schools in Spain}

\section{Políticas públicas educativas sobre migração nos institutos de secundária na Espanha}

\section{Enrique Javier Díez Gutiérrez* - España}

Recibido el 15 de abril de 2011, aceptado el 24 de noviembre de 2011

\section{Resumen}

Objetivo: conocer la percepción, las prácticas y las estrategias que sobre la educación intercultural se están desarrollando en los institutos de educación secundaria de León (España) desde la perspectiva de los equipos directivos y los coordinadores y coordinadoras de convivencia. Metodología: investigación cualitativa, partiendo de una entrevista grupal en profundidad, semi-estructurada, con todo el equipo directivo y los coordinadores de convivencia de todos los institutos. Esta entrevista constó de 35 preguntas abiertas, estructuradas en seis categorías, relacionadas con la situación existente en cada centro respecto a la multiculturalidad, la filosofía y prácticas de educación intercultural que desarrollan, los recursos con los que cuentan, el abordaje curricular y organizativo de la educación intercultural y la formación del profesorado. Resultados: aunque se ha incorporado el concepto de diferencia y lo intercultural en el discurso educativo, el tratamiento dado a la diversidad cultural está muy alejado de lo que se ha configurado en la comunidad científica como educación intercultural, situándose más en el modelo de compensación educativa. Conclusiones: la educación intercultural se asocia esencialmente al incremento de la presencia de inmigrantes extranjeros en el centro educativo, concibiéndose como acciones dirigidas principalmente a los hijos e hijas de personas migrantes extranjeras y de minorías étnicas. La mayor parte de los supuestos conflictos de diferencia cultural tienen que ver con problemas políticos y sociales a los que no se puede responder sólo con soluciones educativas.

Palabras claves: migración interna, inclusión educativa, políticas públicas de migración, políticas escolares frente a migrantes, participación de migrantes, España.

* Ph.D. en Ciencias de la Educación. Profesor Universidad de León. Correo electrónico: enrique.diez@unileon.es 


\begin{abstract}
Objective: this paper aims at identifying the perception, practices and strategies relating to intercultural education that are being developed in secondary schools in León (Spain) from the point of view of school management teams and coordinators responsible for creating an environment of peaceful coexistence between students. Methodology: the methodology used was mainly qualitative. First an in-depth group interview and a semi-structured interview were designed that involved all members of the management teams and the coexistence coordinators from all secondary schools. This interview consisted of 35 open questions that were organized into six categories according to the situation in each school. These categories included multiculturalism, the theory and practices of intercultural education that were being developed, available resources, curricular and organizational approaches to intercultural education and the education of the teachers on the subject. Results: although concepts of difference and interculturalism have been included in the educational discourse, how cultural diversity is treated is far from what the scientific community has defined as intercultural education. It is considered more as a form of compensatory education. Conclusions: intercultural education is essentially linked to the increased number of foreign immigrants in schools and is viewed as a process aimed particularly at the children of foreign migrants or ethnic minority families. Most of the conflicts assumed to arise from cultural difference relate in fact to political and social issues, which cannot be resolved via educational solutions alone.
\end{abstract}

Keywords: migration, educational inclusion, public migration policies, school policies towards migrants, migrant participation, Spain.

\title{
Resumo
}

Objetivo: conhecer a percepção as pratica e as estratégias que sobre a educação intercultural se estão desenvolvendo nos institutos de educação secundariam de Leão (Espanha) desde a perspectiva das equipes diretivas coordenadores de convivência. Metodologia: a metodologia utilizada foi de corte fundamentalmente qualitativo, partindo de uma entrevista grupal em profundidade, semi-estruturada, com toda equipe diretiva e os coordenadores de convivência de todos os institutos. Esta entrevista constou de 35 perguntas abertas, estruturadas em seis categorias, relacionadas com a situação existente em cada centro respeito à multi- culturalidade, a filosofia e praticas de educação intercultural e a formação do professorado. Resultados: porem se tem incorporado o conceito de diferencia e o intercultural no discurso educativo, o tratamento dado à diversidade cultural este muito afastado do que se tem configurado na comunidade cientifica como educação intercultural, situando se mais no modelo de compensação educativa. Conclusões: a educação intercultural se associa essencialmente ao incremento da presencia de imigrantes estrangeiros no centro educativo concebendo se como ações dirigidas principalmente a os filhos e filhas pessoas migrantes e estrangeiras y de minorias étnicas. A Maior parte dos supostos conflitos de diferencia cultural tem que ver com problemas políticos e sociais aos que não se podem responder só com soluções educativas.

Palavras-chaves: migração, inclusão educativa, políticas públicas de migração, políticas escolares frente a migrantes, participação de migrantes, Espanha.

Para citar este artículo:
Díez, E. (junio, 2012). Políticas públicas educativas sobre migración en los institutos de secundaria de España. Ánfora, 19 (32), 15 - 30. Universidad Autónoma de Manizales. ISSN 0121-6538. 


\section{Introducción}

\section{Planteamiento del problema}

Un fantasma recorre Europa: el resurgimiento del racismo institucional plasmado en políticas de gobiernos conservadores como el de Berlusconi en Italia o el de Sarkozy en Francia, y se extiende lenta y sostenidamente por toda la geografía europea. Este discurso está generando una alarma social que se multiplica a través de los medios de comunicación que hacen de amplificadores (Van Dijk, 2010; Olmos, 2010) pues ligan migración y delincuencia a la seguridad; minorías y problemas a la "bajada de nivel" en el sistema educativo; diferencia y amenaza al peligro de la pérdida de identidad. No es de extrañar, por tanto, que este clima se traslade, casi por ósmosis, al entorno educativo.

Los estudios sobre convivencia escolar en España (Calvo, 2008; Instituto de la Juventud, 2008; Díaz, 2008; Olmos, 2010) reflejan una problemática seria de integración y convivencia entre alumnos de diferentes culturas, que se ve agravada con la crisis económica actual. Tomás Calvo Buezas, catedrático de Antropología que dirige el Centro de Estudios sobre Migraciones y Racismo (CEMIRA) de la Universidad Complutense de Madrid, elabora desde 1986 estudios sobre actitudes ante la inmigración y el cambio de valores. Para la encuesta de 2008, consultó a 10.507 escolares, entre 14 y 19 años, de once comunidades autónomas. Según sus resultados, un 53\% de los escolares consideró que debería expulsarse a las personas migrantes en situación irregular ("sin papeles"), y un 51\% pensaba que "quitan trabajo a los españoles", decreciendo actualmente el imaginario romántico de una sociedad mestiza y multicultural.

En este mismo sentido, los datos que aportó el estudio estatal sobre el comportamiento escolar, realizado por el Observatorio Estatal de Convivencia Escolar (dependiente del Ministerio de Educación) entre 23.100 estudiantes de educación secundaria y más de 6.000 profesores y profesoras en 300 centros (Díaz, 2008), concluían que dos de cada tres estudiantes españoles de educación secundaria rechazan trabajar con compañeros gitanos o marroquís.

La convivencia en un contexto educativo de diversidad cultural, con un alumnado heterogéneo, con diferentes intereses y motivaciones, constituye un reto para el profesorado (Carbonell, 2002). Hay que señalar que muchos de los conflictos parten de la configuración de la institución escolar y de su organización (Leyva, 2008), además del contexto social y mediático que afecta profundamente a toda la comunidad educativa construyendo un imaginario poco propicio para el encuentro intercultural.

Esta investigación ha pretendido ser una respuesta inicial a la necesidad detectada por la Concejalía de Bienestar Social del Ayuntamiento de León respecto a las dificultades derivadas del proceso de integración de la población migrante en el ámbito escolar y social de los institutos de enseñanza secundaria de León. Por todo ello, en la 
Universidad de León se ha pretendido contribuir, con esta propuesta, a la mejora de los procesos de integración a través de un proyecto de investigación-acción de Educación Intercultural del alumnado de secundaria.

El objeto de la investigación ha sido conocer, por una parte, la percepción que sobre la educación intercultural tienen los equipos directivos de los institutos de educación secundaria de la ciudad de León y los coordinadores y coordinadoras de convivencia de dichos institutos, así como la opinión y actitud en relación con la atención que el centro concede a la diversidad cultural. Por otra parte, se ha indagado en las prácticas, herramientas y estrategias de educación intercultural que, a juicio de los propios interesados -equipos directivos y coordinadores de convivencia-, se están desarrollando en estas organizaciones, de cara a una mejor convivencia e integración del alumnado en los centros de educación secundaria.

En función de los resultados de la investigación y a partir de la situación detectada, se diseñó un programa de sensibilización en educación intercultural dirigido al alumnado de $1^{\circ}$ de la ESO, a través del conocimiento y el respeto a las diferentes identidades culturales. En este programa se analizan los valores sociales compartidos, se potencian actitudes positivas que eviten la discriminación y sienten las bases para construir una adecuada convivencia intercultural en los institutos de educación secundaria.

\section{Antecedentes y estado actual de la investigación sobre migración y educación intercultural}

Los datos son preocupantes. Hay un sistema educativo sorprendido ante la evolución creciente de la escolarización de alumnado extranjero, sin contar con repuestas que parezcan suficientemente válidas. Hassan Arabi (2001), del Observatorio de la Comunidad de Madrid contra el racismo y la xenofobia, afirma que, en las aulas, los alumnos extranjeros de infantil no presentan muchos problemas para el profesorado, puesto que "no obligan casi ningún cambio en la actitud y el método del profesor o profesora". Con los de primaria, los problemas que se plantean se resuelven rápidamente porque los niños a una menor edad suelen tener mayor capacidad de aprendizaje e integración en el grupo (Rincón y Vallespir, 2010). Los problemas, según el autor, están en la Enseñanza Secundaria Obligatoria ESO. Para él, los alumnos y las alumnas que llegan a España con edades entre 10 y 16 años, con desconocimiento de la lengua, a enfrentar una realidad y un entorno social nuevos son las verdaderas víctimas del actual sistema educativo. Estos alumnos, en la mayoría de los casos, abandonan sus estudios porque se sienten incapaces de seguir el ritmo de sus compañeros y compañeras, son ridiculizados y se creen inferiores al resto del grupo, lo que provoca inseguridad y una autoestima muy baja. Pensemos que, como señala Jordan "los estudiantes minoritarios identifican a los profesores como personas muy significativas, de forma que su autoconcepto está en gran medida determinado por cómo ellos sienten que sus maestros les

Para citar este artículo:
Díez, E. (junio, 2012). Políticas públicas educativas sobre migración en los institutos de secundaria de España. Ánfora, 19 (32), 15 - 30. Universidad Autónoma de Manizales. ISSN 0121-6538. 
perciben" (1988 p. 506). En esta forma, las expectativas previas del profesorado sobre este alumnado pueden convertirse en profecías autocumplidas.

Sin embargo, según estudios recientes (Díaz y Baraja, 1993; Núñez, 2010; Gairín e Iglesias, 2010), muy pocos profesores y profesoras proponen acciones interesantes para atender la diversidad. Una parte del profesorado considera la presencia de inmigrantes en las aulas como un "problema" y una fuente potencial de conflictos, cuando no como un entorpecimiento de la marcha general de la clase. Muchos de los docentes consultados emplean sustantivos como "incapacidad" y "frustración" para describir cómo se enfrentan en sus aulas a una nueva realidad. La frustración y la incapacidad de los profesores y profesoras responden a su falta de preparación para afrontar la realidad multicultural (Soriano, 2008; Leyva, 2008). La mayoría del profesorado que ejerce actualmente en el sistema educativo español no ha sido formado para trabajar en una sociedad culturalmente heterogénea (García y Goenechea, 2009; García, et al., 2010).

Según estas investigaciones, algunos profesores y profesoras refuerzan positivamente a todos sus alumnos y alumnas y sobre todo, a quienes más lo necesitan, mediante métodos adecuados para garantizar que todos obtengan reconocimiento académico. Sin embargo, la mayoría del profesorado elogia y dirige la atención positiva únicamente al alumnado de mayor rendimiento y al que no obstaculiza su rol de docente, mientras que, por el contrario, sólo se dirigen a los alumnos que perciben como más problemáticos para criticar su conducta. En ocasiones, cuando los alumnos problemáticos desempeñan correctamente su tarea, el profesor o la profesora suelen dirigirse a ellos en un tono irónico.

Nos sentimos más identificados con el alumno o alumna cercano, que responde, aunque sea culturalmente distante, del alumno o alumna que aprende su oficio y que se caracteriza sobre todo por su interés, por su sumisión, por su comportamiento no problemático y, en definitiva, por su elevado grado de correspondencia con los atributos de rol que se esperan de quienes siguen fielmente el paso de la institución escolar y su cultura organizativa. Quizá no sea exagerado afirmar que la percepción de la amenaza de la paz en el centro, que aumenta a medida que lo hace la distancia del alumnado respecto al modelo, está estrechamente relacionada con la percepción de la amenaza del orden social con que se produce la estereotipia de los grupos étnicos más distantes culturalmente, en ocasiones, pero más próximos en la realidad cotidiana. Así la percepción claramente complaciente que se tiene del alumnado asiático suele hacer referencia a su capacidad de esfuerzo, a su motivación y, sobre todo, a su disciplina. Su bajo rendimiento académico, cuando se da, suele atribuirse normalmente a un pobre conocimiento de la lengua de acogida, se considera fácilmente subsanable e incluso se ve en ello frecuentemente un reto personal (Terrén, 2004, pp. 40-41).

La investigación sobre educación intercultural (García y Escarbajal, 2009) demuestra que:

Para citar este artículo:
Díez, E. (junio, 2012). Políticas públicas educativas sobre migración en los institutos de secundaria de España. Ánfora, 19 (32), 15 - 30. Universidad Autónoma de Manizales. ISSN 0121-6538. 
La norma que fija la administración, si bien declara la necesidad de fomentar modelos interculturales, se decanta finalmente por el modelo de compensatoria, y aunque manifiesta que considera la diferencia como riqueza, parece mantener arraigada la idea del déficit. De manera que, a pesar de que la norma defiende explícitamente posiciones ideológicas de integración, opta por modelos de compensatoria que están vinculados a ideologías marginadoras (Rojo, 2003, p. 231).

Esta misma autora reconoce que en la mayoría de los centros se encuentra arraigado el modelo de compensatoria en la práctica educativa; que ni la legislación ni los centros han iniciado la transformación de los currículos, de forma que se combatan los prejuicios étnicos y que los contenidos, los estilos educativos y los materiales didácticos representen y se adapten a una audiencia plural y heterogénea; que es pequeñísimo el porcentaje de alumnos al que van dirigidos estos programas de compensatoria, que pasan a bachillerato; que los centros educativos constituyen comunidades monolingües, a pesar del multilingüismo de los estudiantes. Constata además que ni la administración impulsa el mantenimiento y uso de estas lenguas, ni el profesorado las incorpora a las prácticas educativas (García, et al., 2010, p. 490). Esta orientación se debe a que el auge de los estudios sobre educación intercultural se inició en España en la década de los ochenta, con la visibilización de la migración.

Esto va a ser determinante para el modelo de respuesta por el que se opta, al desarrollarse más como reacción a la presencia de inmigrantes en las aulas a través de medidas compensatorias, que como una revisión de la inadecuación de los supuestos homogeneizadores y etnocéntricos sobre los que se asienta el propio sistema (García y Goenechea, 2009, pp. 87-88).

La solución que se ha planteado por las administraciones educativas es enviar profesorado de compensatoria a los centros donde la concentración de inmigrantes es mayor para atender a estos escolares (Heckmann, 2008, p. 59). Dicha solución no hace más que agrandar el problema y seguir la política del avestruz. Primero, porque esta política favorece guetos de población inmigrante en determinados barrios o colegios, lo que dificulta la integración de los migrantes en la sociedad receptora. Y, segundo, porque la respuesta a la diversidad y al multiculturalismo con especialistas hace que el profesorado ordinario eluda el asunto al delegar sus responsabilidades a los expertos o al profesorado de compensación educativa o de apoyo (García, et al., 2010, p. 489).

Las propuestas de las acciones educativas de compensatoria, habitualmente van en la línea de crear "programas específicos de aprendizaje". El problema es que si se educa al alumnado inmigrante por separado, se restan oportunidades para fomentar su competencia social, para cooperar con otros alumnos y alumnas diferentes y se favorece la conformación de entornos sociales potencialmente fragmentados (Gorski, 2009). Educar por separado, para después convivir juntos, parece un contrasentido (Jiménez, 20 2010, p. 432).

Para citar este artículo:
Díez, E. (junio, 2012). Políticas públicas educativas sobre migración en los institutos de secundaria de España. Ánfora, 19 (32), 15 - 30. Universidad Autónoma de Manizales. ISSN 0121-6538. 


\section{Metodología}

La peculiaridad de los fenómenos que estudia el campo de las ciencias sociales obliga a utilizar diferentes métodos en su investigación. Por tratarse de conocer, develar y analizar buenas prácticas de educación intercultural no es pertinente dedicarse exclusivamente a la valoración de resultados. Los procesos educativos son complejos. Intervienen en ellos multitud de factores interrelacionados cuyo aislamiento sólo puede conseguir la distorsión del conocimiento pretendido.

A veces, ese afán por mantener un supuesto "rigor" en la investigación conduce a la selección, consciente o inconscientemente, de las variables cuantificables u operativas, con el doble peligro, por un lado, de tomar en consideración los aspectos menos relevantes y, por otro, llegar a conclusiones sesgadas, erróneas y engañosas.

Pero hay un mayor peligro. Desde la perspectiva cuantitativa, se tiende a suprimir de la investigación todo aquello que no va a ser medido, es decir, lo difícilmente cuantificable, o al menos se relega a un segundo plano en la práctica.

La complejidad de los fenómenos educativos es de tal magnitud que difícilmente pueden ser investigados a través de mediciones. Cuando en aras de una supuesta "mayor cientificidad", diseccionamos la realidad, descontextualizamos los fenómenos educativos y les atribuimos valores numéricos, a fin de adaptarlos a una metodología cuantitativa, es fácil que hayamos forjado una imagen de esa realidad irreconocible por los que en ella están inmersos y, por tanto, incapaz de producir conocimiento válido para la comprensión, la reflexión y la mejora de la intervención educativa o social (Fernández y Santos, 1992, p. 23).

Se entiende que esta opción hacia una metodología de carácter cualitativo es un planteamiento cada vez más común entre las investigadoras y los investigadores de ciencias sociales (etnografía, antropología, psicología, pedagogía, sociología, historia, etc.). John Van Maanen (1983) lo atribuye al descrédito generalizado de las técnicas cuantitativas al ver la escasa proporción de varianza que consiguen explicar adecuadamente mediante modelos estadísticos; al carácter esotérico y de poco contenido social real de las variables claves del método cuantitativo; a la poca comparabilidad real de las investigaciones cuantitativas; a la tendencia hacia las investigaciones expostfacto, ya que es muy pobre la validez predictiva de la mayoría de los estudios cuantitativos; a la sofisticación matemática e informática tan alta que impide el acceso a este campo más que a unos pocos especialistas; y porque la complejidad relacional del análisis multivariado paraliza cualquier intento de orientación práctica a la toma de decisiones o a la investigación de una situación concreta.

Pero no se argumenta a favor de este tipo de investigación de carácter eminentemente cualitativo por imposibilidad técnica de un tipo de investigación cuantitativa en la esfera educativa. De hecho, es la más frecuente en estos últimos años. Se entiende

Para citar este artículo:
Díez, E. (junio, 2012). Políticas públicas educativas sobre migración en los institutos de secundaria de España. Ánfora, 19 (32), 15 - 30. Universidad Autónoma de Manizales. ISSN 0121-6538. 
que una opción metodológica supone una opción política y ética sobre la práctica: práctica no sólo de conocimiento, de proceso de aprendizaje-enseñanza, sino práctica que implica un proceso de transformación o de mantenimiento de la realidad. Porque lo que, en definitiva, se busca con esta investigación es que tenga utilidad y sirva a las comunidades educativas en su práctica cotidiana profesional.

Por eso, en la fase de investigación propiamente dicha, se ha utilizado una metodología cualitativa -aunque se han recabado también algunos datos de corte cuantitativo que complementan la información-, puesto que se trata de describir, comprender y explicar fenómenos educativos (Velasco y Díaz, 1997). Aunque la opción es de tipo cualitativo, no obstante, siguiendo la propuesta de Cook y Reichardt (1986), es necesario superar la falsa disyuntiva o enfrentamiento entre la utilización de métodos cuantitativos (aquellos que buscan a través de los criterios de medida datos cuantificables y medibles en porcentajes) y métodos cualitativos (aquellos que buscan la información que subyace a la realidad y a los fenómenos y los procesos, y de los criterios que la configuran, y que ayudan a analizarla, comprenderla y conocerla mejor para su intervención).

La negociación entre ambas metodologías es lo que se ha llamado "triangulación". De ahí que la metodología cualitativa haya sido completada con datos de corte cuantitativo, que son dos formas de aproximación a una misma realidad que, más que excluirse, llegan a integrarse, progresando cada una gracias a la otra, pues las aportaciones de una complementan las posibles carencias de la otra.

Respecto a la fase de acción de los diferentes institutos de enseñanza secundaria, se seleccionaron dos de ellos, representantes de la realidad de los IES de León, en los que se trabajó con dos de los grupos de primer año de la ESO. Las variables que se tuvieron en cuenta para seleccionar los IES fueron: presencia de alumnado migrante y de minorías; tamaño del centro; su ubicación y preocupación por el tema de la comunidad educativa. Fueron así seleccionados un IES con una escasa presencia de población migrante/minorías étnicas -0,5\%-; un gran centro - 1.600 alumnos y alumnas-, situado en el núcleo urbano central de la ciudad y con percepción de baja preocupación de la comunidad educativa por este tema; un segundo IES con numerosa presencia de población migrante, aglutinada principalmente en uno de los primeros de la ESO, concretamente el "no bilingüe"; y un centro mediano/pequeño situado en la periferia de la ciudad y con percepción de alta preocupación de la comunidad educativa por esta temática.

Con cada grupo de primero de la ESO de las dos IES, se trabajaron tres sesiones de sensibilización, durante las horas de tutoría con la presencia y participación de las tutoras, con un planteamiento eminentemente práctico, que utiliza el Taller como base para el desarrollo de las diferentes dinámicas/actividades previstas en el aula. Se partió de las experiencias del grupo y se provocaron situaciones que les ayudaron a experimentar

Para citar este artículo:
Díez, E. (junio, 2012). Políticas públicas educativas sobre migración en los institutos de secundaria de España. Ánfora, 19 (32), 15 - 30. Universidad Autónoma de Manizales. ISSN 0121-6538. 
el aspecto que se estaba trabajando. De esta manera, se activó en el alumnado el sentir, el pensar $y$, posteriormente, el actuar.

La estructura de cada taller se iniciaba con la presentación de la temática, utilizando para ello dinámicas de animación. A partir de ahí, se desarrollaron los contenidos desde el conflicto cognitivo y, con base en el aprendizaje cooperativo, se realizaron actividades específicas para cada temática que permitieran vivenciar en el alumnado su propia experiencia las problemáticas existentes en la integración. El cierre del taller recapituló las ideas principales entre todo el alumnado y se valoró su utilidad.

Simultáneamente a los talleres, se adelantó la elaboración colectiva de un calendario intercultural con el apoyo del profesorado de "educación plástica y visual". Este calendario fue una representación visual de los diferentes aspectos que reflejan el trabajo realizado con el alumnado a través de la sensibilización en los IES y buscaba una repercusión social. Las imágenes de la portada y las de los doce meses son dibujos de los alumnos de los dos IES. Cada día del mes tiene una frase, actividad o recordatorio de fecha importante relacionada con la interculturalidad. Cada una de las actividades propuestas para que los estudiantes realizaran en el aula, en sus hogares o con sus iguales, está vinculada con una serie de códigos que expresan la competencia intercultural que se trabaja. Al finalizar el calendario, en la última página se aportan los conocimientos básicos, relativos a las competencias interculturales que se abordan con las diferentes actividades propuestas en cada mes, para la comprensión de la utilidad del calendario Intercultural en el aula 2011.

\section{Instrumentos de recogida de datos}

La recolección de datos se hizo mediante una entrevista grupal en profundidad, semi-estructurada, con todos los componentes del quipo directivo y los coordinadores y coordinadoras de convivencia en los diferentes IES de la ciudad de León. El objetivo de esta entrevista fue conocer la problemática en cada centro, la forma de actuar de éstos ante los conflictos que se plantean y la incorporación a las IES, según un enfoque transversal de la educación intercultural como base para lograr una idónea convivencia escolar.

El diseño de la entrevista en profundidad se basa en los indicadores para la convivencia escolar de los centros educativos de Castilla y León (Consejería de Educación de la Junta de Castilla y León, 2010) y en diversos instrumentos: "Evaluación de la participación de padres, profesores y alumnos en los centros docentes" (Aguado, Bernal, Bernat, García, Ramos y Sabirón, 1988), "Entrevista sobre el prejuicio étnico" (Díaz y Baraja, 1994), "Encuesta sobre diversidad cultural e igualdad escolar" (Aguado, 1999).

Este instrumento recaba información sobre las opiniones y actitudes del propio equipo directivo, del profesorado del centro y de la comunidad educativa del instituto, 
en relación con la diversidad cultural presente en el centro y en la dinámica cotidiana de interacción entre ellos. Contiene diversas preguntas encaminadas a recabar información sobre aspectos organizativos y curriculares que inciden especialmente en la forma de dar respuesta a la diversidad cultural del centro, dedicando especial atención a conocer posibles estrategias de adaptación e individualización de la enseñanza, servicios de apoyo y utilización de recursos específicos. Asimismo, una parte de las cuestiones planteadas pretenden averiguar cuáles son las categorías según las cuales se describen las diferencias culturales y el conocimiento que se tiene sobre los diferentes grupos identificados. Por último, se pregunta acerca de la opinión en torno a cómo habría que abordar la diversidad cultural en el medio escolar.

\section{Características de la muestra}

Han participado en la investigación todos los equipos directivos de los institutos de educación secundaria de la ciudad de León y los coordinadores y coordinadoras de convivencia de dichos institutos. La muestra se compone de nueve institutos de educación secundaria, en los que se realizó una entrevista grupal a cuatro personas (tres componentes del equipo directivo y el coordinador o coordinadora de convivencia), por lo que la muestra final son 36 personas.

En cuanto a la población participante en el programa de sensibilización, la muestra se compone de dos IES, en cada uno de ellos dos grupos de primer año de la ESO y en total 87 alumnas y alumnos, con los que se desarrollaron las dinámicas en los talleres de sensibilización y las cuatro tutoras de los grupos y el jefe y la jefa de estudios de cada Instituto que asistieron a los talleres.

\section{Resultados}

Aunque se ha incorporado el concepto de diferencia y de interculturalidad en el discurso educativo, el tratamiento dado a la diversidad cultural está muy alejado de lo que se ha configurado como educación intercultural. Esta se sigue asociando al incremento de la presencia de inmigrantes extranjeros en el centro educativo. Aunque alguna de las personas entrevistadas enfocaba tímidamente la educación intercultural desde una perspectiva que pretende involucrar a todos los participantes en el proceso educativo y todas las dimensiones del centro. El enfoque más común buscó plantear actuaciones educativas específicas para diversos grupos culturales, con el fin de articular medidas de compensación para que el alumnado de minorías y migrante aprendiera el idioma y lograra integrar las normas y costumbres del país y adaptarse a la sociedad de acogida. Sólo en un IES podemos afirmar con claridad que hay presente un auténtico enfoque intercultural en la mayor parte del curriculum y de la organización y funcionamiento del centro.

Se siguen adelantando estrategias potencialmente discriminatorias relacionadas con

24 los criterios de clasificación o agrupamiento del alumnado, no sólo por zonas, centros

Para citar este artículo:
Díez, E. (junio, 2012). Políticas públicas educativas sobre migración en los institutos de secundaria de España. Ánfora, 19 (32), 15 - 30. Universidad Autónoma de Manizales. ISSN 0121-6538. 
(públicos y concertados), sino también por aulas mediante las opciones de agrupación del alumnado. Se comprueba una distancia significativa entre las propuestas normativas que se recogen en los documentos oficiales de los institutos y las informaciones manifestadas por las personas entrevistadas. Se detecta una dificultad para reconocer las necesidades específicas y los aportes de los grupos más diferenciados en el centro.

Se han identificado modelos diferenciados de actuación educativa en función del tratamiento dado a la diversidad cultural. Las diferencias entre centros en cuanto a su grado de ajuste al modelo intercultural se explican por las dimensiones orientación, enfoque y atención a la diversidad; actividades y estrategias interculturales; implicación de la dirección; estilo de enseñanza del profesorado; y características y utilización del material.

Respecto a las características y necesidades educativas señaladas (población migrante y de etnia gitana) los equipos directivos y de orientación señalan que suelen presentar desfases curriculares y, en algunos casos, desconocimiento de la lengua oficial y vehicular de los aprendizajes escolares, lo que, para el profesorado, supone un problema. Las soluciones más habituales son adaptaciones curriculares específicas, a cargo del profesorado de compensatoria, que suele intervenir en estos casos en espacios separados durante algunas horas lectivas, de forma simultánea a las clases.

Se considera en general que tienen baja motivación por el tipo de aprendizaje escolar dado que "no se sienten identificados con nuestro sistema educativo" (señalan especialmente a magrebíes y gitanos) o "tienen un dominio del español 'de la calle', pero habitualmente no saben escribirlo o leerlo". Los entrevistados aluden a la dificultad que encuentra el alumnado para comprender el lenguaje académico utilizado continuamente en el contexto escolar. Algunos centros también se refieren a diferencias de edad: "los más jóvenes están menos motivados que el alumnado de cursos con un nivel superior"; o de sexo: "en general, las chicas tiene más hábito de trabajo que los chicos, y tiene más aspiraciones personales que les motiva para esforzarse estudiando, mientras que los chicos están más abandonados".

\section{Conclusiones}

Las familias de la población migrante y de minorías suelen presentar bajas expectativas respecto a las posibilidades de éxito escolar de sus hijos y sus hijas y a la utilidad de la formación reglada. Se tiende a considerar que son familias poco colaboradoras y participativas y que no dan toda la importancia necesaria a la educación de los niños y las niñas -aunque reconocen que esto pasa con la mayoría de las familias-. Con estas familias suele haber problemas de comunicación por dificultades de comprensión de la lengua o dificultades de encuentro, dados los horarios laborales de los padres y las madres. Esto no obsta para que también se mencionen casos concretos de familias de población migrante o de etnia gitana muy participativas que contribuyen a una mejor 
comprensión de su cultura, colaboran con el centro, como las familias Magrebíes en el IES Padre Isa, o familias gitanas en el IES García Bellido.

En varios centros, se considera que el alumnado de etnia gitana es el que presenta un mayor número de sanciones o llamadas de atención por conductas anómalas en el aula/centro y por las dificultades que encuentran de adaptación a la metodología "clásica" de comportamiento en el aula. Uno de los Institutos se planteó la necesidad de incorporar una metodología docente activa que tenga como base el aprendizaje significativo del alumnado, para lograr un aprendizaje real con este colectivo.

Quien se encarga de la educación intercultural suele ser el coordinador de convivencia, el jefe de estudios o el orientadora. No se encuentra en los IES analizados una figura específica dedicada a tratar los temas relacionados con la educación intercultural, aunque sí está recogido normativamente en la educación para la igualdad, de modo que algunos IES lo tienen incorporado bajo el marco de la llamada "convivencia escolar". Cabe destacar el IES García Bellido, que dispone de una profesional encargada de trabajar el tema de "Igualdad" de sexos y de origen cultural.

En los IES se tiene la percepción de que toda la comunidad educativa se implica "de forma difusa" en estos temas con buena voluntad, aunque en la realidad la participación suele referirse a casos específicos, con participación especial del profesorado sensibilizado. El profesorado, a excepción de algún IES con una dinámica global de trabajo por la diversidad o de convivencia, participa escasamente y cuando lo hace se debe a una motivación personal y a una sensibilidad hacia la interculturalidad, o por una finalidad instrumental al dedicar sus "horas sobrantes" al apoyo escolar.

Algunos aportes manifestados para mejorar la atención a la interculturalidad son:

1. Mayor formación del profesorado en educación intercultural.

2. Reducción del número de alumnos por profesor y por grupo.

3. Estabilidad de las plantillas del profesorado.

4. Libros de texto y materiales curriculares que incorporen una visión más intercultural.

5. información traducida a las lenguas de origen de las familias inmigrantes, sobre el proceso de matrícula, sobre las características esenciales del sistema educativo o sobre los requisitos para solicitar una beca de comedor o ayudas al estudio.

6. Crear un espacio en la biblioteca del centro, destinado a literatura y ensayos en las lenguas de origen de la población migrante.

7. Destinar tiempos al trabajo de habilidades sociales, de comunicación y de resolución de conflictos en las materias de lengua, educación para la ciudadanía, tutoría y otras. 
Estas propuestas de nada servirían si no van acompañadas de políticas sociales favorables. Se entiende que si no se aborda el contexto social en el que se enmarca la acción educativa de los centros, no tiene ningún sentido las proclamas teóricas de educación intercultural ni las prácticas que se lleven a cabo en ellos, pues la interculturalidad no es "un problema" estrictamente escolar, sino una cuestión de carácter sociopolítico (Besalú, 2002). La mejor educación intercultural es el reconocimiento social, económico y político pleno de las minorías culturales. No se puede responder a los problemas políticos y sociales sólo con soluciones educativas.

Como explica Innerarity (2011), la clave del asunto sigue siendo política, de derechos y no tanto de diversidad cultural. Esta es la tesis de Sami Naïr (2010) que previene contra la "culturalización" del fenómeno que reduce la interculturalidad a la protección de las diferencias culturales. Su enfoque es, por el contrario, el de los derechos humanos, donde se considera que el proyecto intercultural no es tanto en el terreno cultural como en la práctica social (en movilidad, derechos, participación e igualdad personal). Naïr propone abordarlo como una cuestión política, en el sentido radical del término inmigración. Esto significa que las personas migrantes deben estar en el centro de las políticas públicas y no en su periferia, como una cuestión de asistencia a colectivos marginales o vulnerables, o como aspectos tangenciales que se tratan en el ámbito educativo, sanitario o social. Es necesario que Europa deje de considerar la migración como un problema de seguridad, de fronteras y de policía, y lo gestione como un asunto de derechos y ciudadanía, en condiciones de igualdad.

Las condiciones de ese proyecto intercultural son, sobre todo, económicas, jurídicas y políticas. La mayor parte de los conflictos de diferencia cultural tiene que ser abordada desde la perspectiva previa de conflictos de intereses sobre la distribución de la riqueza, la participación en el poder, las condiciones para esa participación y la distribución, comenzando por la reparación de las situaciones heredadas de discriminación y dominación.

\section{Referencias}

Aa.vv. (2005). Inmenso estrecho. Cuentos sobre inmigración. Madrid: kailas.

Aa.vv. (2010). Libro blanco de la educación intercultural. Madrid: fete-ugt.

Aguado, T. (1999). Diversidad cultural e igualdad escolar. Un modelo para el diagnóstico y desarrollo de actuaciones educativas en contextos escolares multiculturales. Madrid: CIDE.

Aguado, T. (2003). Pedagogía intercultural. Madrid: McGraw-Hill.

Aguado, T. (2007). Racismo, qué es y cómo se afronta. Madrid: Pearson - Prentice Hall.

Aguado, T. y Olmo, M. (coord.). (2009). Educación intercultural. Perspectivas y propuestas. Madrid: Ramon Areces.

Para citar este artículo:
Díez, E. (junio, 2012). Políticas públicas educativas sobre migración en los institutos de secundaria de España. Ánfora, 19 (32), 15 - 30. Universidad Autónoma de Manizales. ISSN 0121-6538. 
Aguado, T.; Bernal, J.; Bernat, A.; García, E. y Sabiron, E. (1988). Evaluación de la participación de padres, profesores y alumnos en los centros docentes. Zaragoza: Universidad de Zaragoza.

Alemán, J. et al. (2009). Los otros entre nosotros: alteridad e inmigración. Madrid: Círculo de Bellas Artes.

Arabi, H. (2001). La educación intercultural ¿un nuevo frente en la formación del profesorado? Ponencia presentada en las Jornadas sobre Interculturalidad: Educar en las Aulas. Retos del siglo XXI celebradas en Murcia en diciembre de 2001. Recuperado de http://www.aulaintercultural.org/article.php3?id_article=39]

Banks, J. (2009). The Routledge International Companion to Multicultural Education. New York: Routledge.

Bartolomé, M. (2002). Identidad y ciudadanía: un reto a la educación intercultural. Madrid: Narcea.

Belsué, K. (2009). El rechazo invisible: integración que discrimina. En Catalá, M. Miradas y voces de la inmigración (pp. 65-77). Barcelona: Montesinos.

Besalú, X. (2002). Diversidad cultural y educación. Madrid: Síntesis.

Besalú, X. (2010). La Educación Intercultural y el Currículo Escolar. Ponencia en el Ier Congreso Internacional en la Red sobre Interculturalidad y Educación. Recuperado de http://letra25. com/ediciones/cii-viernes1/besalu/].

Calvo, T. (1989). Los racistas son los otros. Gitanos, minorías y Derechos Humanos en los Textos Escolares. Madrid. Popular.

Calvo, T. (2008). Actitudes ante la Inmigración y Cambio de Valores. Madrid: Centro de Estudios sobre Migraciones y Racismo UCM.

Carbonell, F. (2002). Educación Intercultural: principales retos y requisitos indispensables. Cooperación Educativa, 65, 63-68.

Catalá, M. (Coord.). (2009). Miradas y voces de la inmigración. Barcelona: Montesinos.

Connell, R. (1997). Escuelas y justicia social. Madrid: Morata.

Consejería de Educación de la Junta de Castilla y León. (2010). Indicadores para la Convivencia escolar de los centros educativos de Castilla y León. Recuperado de http://www.educa.jcyl.es/edu$\mathrm{cacyl} / \mathrm{cm} /$ convivencia/images?locale=es_ES\&textOnly=false\&idMmedia=175035]

Cook, T. y Reichardt, Ch. (1986). Métodos Cualitativos y cuantitativos en investigación evaluativa. Madrid: Morata.

Díaz, M. (Dir). (2008). Estudio Estatal sobre la convivencia escolar en la Educación Secundaria Obligatoria. Madrid: UCM. Recuperado de http://www.oberaxe.es/files/datos/4880737908ab1/ MECavance_resultados_2008.pdf].

Díaz, M. y Baraja, A. (1994). Interacción educativa y desventaja sociocultural. Un modelo de intervención para favorecer la adaptación escolar en contextos inter-étnicos. Madrid: CIDE.

Díez, E. (2005). La educación intercultural en una sociedad mestiza. Opciones Pedagógicas, 31, 70-95.

Para citar este artículo:
Díez, E. (junio, 2012). Políticas públicas educativas sobre migración en los institutos de secundaria de España. Ánfora, 19 (32), 15 - 30. Universidad Autónoma de Manizales. ISSN 0121-6538. 
Díez, E. (2009). Globalización y educación crítica. Bogotá: Ediciones Desde Abajo.

Essomba, M. (2007). Estrategias de innovación para construir la escuela intercultural. En Álvarez, J., y Batanaz, L. Educación intercultural e inmigración. De la teoría a la práctica (pp. 177212). Madrid: Biblioteca Nueva.

Essomba, M. A. (2008). 10 ideas clave. La gestión de la diversidad cultural. Barcelona: Graó.

Fernández, J. y Santos, M. (1992). Evaluación cualitativa de programas de educación para la salud: una experiencia hospitaliaria. Málaga: Aljibe.

Flecha, R. (2010). Establecer agrupaciones heterogéneas con recursos reubicados. En Aa.Vv. Libro blanco de la educación intercultural (pp. 35-36). Madrid: FETE-UGT.

Gairín, J. e Iglesias, E. (2010). El Programa Curricular en contextos escolares con fuerte presencia de los alumnos de familia inmigrante. Bordón 62(1), 61-75.

García, J. y Goenechea, C. (2009). Educación Intercultural. Análisis de la situación y propuestas de mejora. Madrid: Wolters Kluwer.

García, J.; Sánchez, P.; Moreno, I. y Goenechea, C. (2010). Estudio del sistema y funcionamiento de las aulas de enlace de la Comunidad de Madrid: de la normativa institucional a la realidad cotidiana. Revista de educación, 352, 473-493.

García, A. y Escarbajal, A. (Coord.). (2009). Pluralismo sociocultural, educación e interculturalidad. Madrid: @becedario.

García, M. y otros. (2010). Estrategias bilingües e interculturales en familias transmigrantes. Revista de educación, 352, 289-308.

Gorski, P. (2009). Intercultural education as social justice. Intercultural Education, 20(2), 87-90.

Heckmann, F. (2008). Education and Migration, strategies for integrating migrant children in European schools and societies. A synthesis of research findings for policy-makers. Informe presentado ante la Comisión Europea en nombre de la red de expertos Nesse. Recuperado de http://www. nesse.fr/nesse/activities/reports/activities/reports/education-and-migration-pdf]

Instituto de la Juventud. (2008). Sondeo de opinión y situación de la gente joven ( $2^{\mathrm{a}}$ encuesta 2008. Jóvenes e Inmigración). Madrid: Observatorio de la Juventud en España.

Innerarity, D. (2011). Verdadera política de inmigración. El País, 12 de febrero de 2011.

Jiménez, R. (2010) ¿Diálogo o confrontación de culturas en Ceuta?: un estudio de caso en un Instituto de Educación Secundaria. Revista de educación, 352, 431-451.

Jordán, J. (1999). El profesorado ante la educación intercultural. En Essomba, M. (Coord.). Construir la escuela intercultural. Reflexiones y propuestas para trabajar la diversidad étnica y cultural, (pp. 65-73). Barcelona: Grao.

Jordan, J. (2007). Educar en la convivencia en contextos multiculturales. En Soriano, E. Educación para la convivencia intercultural (pp.59-94). Madrid: La Muralla.

Kincheloe, J. y Steinberg, S. (1999). Repensar el multiculturalismo. Barcelona: Octaedro.

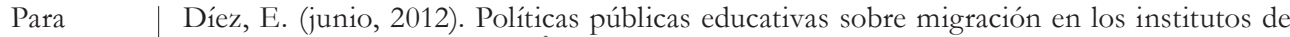


Leyva, J. (2008). Interculturalidad, gestión de la convivencia y diversidad cultural en la escuela: un estudio de las actitudes del profesorado. Revista Iberoamericana de Educación, 46 (2), 1-14.

López, M. (2006). Cultura de la diversidad, cultura de la inclusión: educar para construir una escuela sin exclusiones. Actas de las XVI Jornadas Municipales de Psicopedagogía "L'Ecola que inclou”, 11-52.

Naïr, S. (2010). La Europa mestiza. Inmigración, ciudadanía, codesarrollo. Madrid: Galaxia Gutenberg.

Olmos, A. (2010). Construcción discursiva del alumno inmigrante extranjero en Andalucía: el otro en nuestras escuelas. Revista de Educación, 353, 469-493.

Rincón, J. y Vallespir, J. (2010). El tratamiento de la interculturalidad en los centros de primaria de las Islas Baleares, según las programaciones generales anuales: el Plan de Acogida Lingüística y Cultural. Revista de Educación, 353, 415-441.

Rojo, M. (Dir.). (2003). ¿Asimilar o Integrar? Dilema ante el multilingüismo en las aulas. Madrid: MECD-CIDE.

Santos, M. (2008). La pragmática de la cooperación o cómo mejorar la gestión de la diversidad cultural y de la convivencia en las aulas. En Vera, J. (Coord.): Diversidad, convivencia y educación desde el conflicto, (pp. 55-75). Madrid: Fundación SM.

Soriano, E. (2008). Formación del profesorado para la educación intercultural. En Vera, J. (Coord.) Propuestas y experiencias de educación intercultural, (pp. 57-84). Madrid: Fundación SM.

Van Dijk, T. (2010). Racismo y la Prensa: Análisis Crítico del Discurso. Ponencia en el Auditórium del Edificio Centro de Innovación Docente de la Facultad de Filosofía y Humanidades de la Universidad Austral de Chile el 3 de enero de 2010.

Van Maanen, J. (1983). Qualitative methodology. Beverly Hills, CA: Sage Publications.

Velasco, M. y Díaz, A. (1997). La lógica de la investigación etnográfica. Madrid: Nona.

Para $\mid$ Díez, E. (junio, 2012). Políticas públicas educativas sobre migración en los institutos de les. ISSN 0121-6538. 